\title{
LA AUTODETERMINACIÓN DEL YO-INDIVIDUO Y SUS DOS SERIES EN EL $\$ 17$
}

\author{
THE SELF-DETERMINATION OF THE I- \\ INDIVIDUAL AND ITS TWO SERIES IN \$17
}

JaCinTO Rivera De Rosales

UNED

Nos interesa aquí hacer una presentación esquemática del largo y complejo $\$ 17$, sobre todo de su segunda parte. Desde el $\$ 14$ está planteada la tarea de toda esta segunda parte de la WLnm, hasta el final: cómo el querer puro se convierte en un querer empírico, se sensibiliza, de manera que es para el Yo, o sea, que se le hace presente al Yo y él toma conciencia de sí, de su querer, de qué es él, como individuo y como comunidad. El querer puro había sido alcanzado en el $\$ 13$ y expuesto como condición suprema de toda conciencia. En él, querer y conocer, libertad y limitación, se coimplican mutuamente, es decir, no se daría lo uno sin lo otro, pues la voluntad pura se limita a sí misma mediante su ley moral a fin de preservarse justamente en su modo de ser; esta limitación provoca el sentimiento del deber, de no-tener-el-derecho (Nichtdürfens) a ir más allá aunque así se desee. Lo determinable de esa autodeterminación (pues toda determinación de la actividad es comprendida como un paso de lo determinable a lo determinado, según hemos visto repetidas veces a lo largo del proceso de la WLnm) es el mundo de los seres racionales finitos, la intersubjetividad, el reino de fines del que habla Kant, del cual sale y se desprende el individuo. Esto por ahora sólo lo ve el filósofo. El Yo lo comprenderá después, y lo ha de hacer para configurarse como un Yo, pues él es Yo si sabe de sí; en el proceso de sensibilización de su querer, se percibirá como un individuo determinado dentro de una comunidad de seres humanos (lo determinable), la razón-nosotros (también sensibilizada) que lo hace posible ( $\$ 16,18$ 
y 19). Este es el proceso que se inicia a partir del $\$ 14$ y en el que hemos de insertar y comprender el $\S 17$.

En los $§ \S 14$ y 15 Fichte nos avanza que dicho proceso de sensibilización del querer puro se basa primeramente en la limitación ideal de nuestra reflexión, una limitación que consiste en que no puede captar de un golpe todo lo que yo soy, la totalidad de mi querer puro, sino que ha de ir de una parte a otra, de parte en parte, discursivamente. La reflexión o actividad ideal del Yo es ciertamente libre de escoger en qué parte se fija, qué querer concreto va a satisfacer (Befriedigung), pero sólo puede comprender esa parte distinguiéndola de las demás, estableciendo diferencias y límites, entendiéndola como siendo ella y no las otras, concentrándose en un punto y atendiendo a un momento de mi querer. Eso explaya necesariamente el querer puro en una multiplicidad de actos de querer (empíricos) extendidos en el tiempo; por ese pensar analítico surge el tiempo y la multiplicidad (das Mannigfaltige). En segundo lugar la voluntad pura se objetiva o queda intuida en un cuerpo, el propio cuerpo orgánico de cada uno de nosotros $(L e i b)$, que aquí ya no aparece como un mero instrumento de la libertad (cuerpo articulado), sino como la propia libertad bajo la forma de la intuición empírica espacio-temporal, como su sistema de la sensibilidad u órgano externo (cuerpo sensible, capaz de sentir), abierto al mundo. Esto constituye una limitación originaria que el Yo no produce realiter (el Yo no es un Dios creador ex nibilo del mundo, ni de sí mismo en la medida en que es parte de la naturaleza ${ }^{1}$ ), sino que sólo la reproduce idealiter en su sentido u órgano interno (percepción). Pero al mismo tiempo ella tiene que ser consecuencia de una limitación llevada a cabo idealiter por el propio Yo, como se nos dice en el $\$ 15$, de un autolimitarse del Yo, pues en caso contrario esa limitación originaria no sería para el Yo, estaría dada pero no elaborada por él. Y así es: yo estoy originariamente limitado por la ley (en el fondo moral) de mi propio querer y ésa es mi individualidad ${ }^{2}$, y por ello me convierto en un órgano externo: el cuerpo como sentido (externo). Si yo no me limitara, no habría para mí un mundo. Al limitarme me hago receptivo, me hago mundo y conciencia de mundo (Fichte usa la metáfora de salir al mun-

\footnotetext{
${ }^{1}$ WLnm-K, 496.

${ }^{2}$ Fichte piensa la ley moral no sólo en su aspecto universal, como Kant, sino también en su vertiente individual: cada uno tenemos nuestra Bestimmung, nuestra determinación moral, nuestro quehacer propio en este mundo, nuestro trabajo específico (según los tiempos y las capacidades) en pro de la realización plena de la razón en el mundo.
} 
do), acepto que exista otra realidad además de mí, lo cual es en el fondo una tarea moral para un ser libre.

Con ello tendríamos las condiciones negativas para la sensibilización del querer puro: la limitación, tanto de la acción ideal (de la reflexión) como de la real (órgano externo). Pero eso no basta, sólo de algo positivo puede resultar la limitación que el individuo lleva a cabo en su voluntad (algo positivo ha de ser lo primero). Necesitamos un querer positivo, un acto de querer, el cual, ya que el individuo no ha realizado aún ese acto positivo de querer, éste le tiene que venir dado de fuera. En virtud de esta carencia (Mangel) constitutiva suya, el individuo se abre a la comunidad como una condición de posibilidad que le constituye en su propio ser. Ese acto positivo de querer empírico le viene gracias a la exhortación o requerimiento o solicitación (Aufforderung) del otro a que actúe desde sí, desde su actividad libre, en un sentido o en otro, que haga algo. Eso es lo que se nos explica en el $\S 16$ y se nos recuerda al inicio del $§ 17:$ : Mi individualidad sale de la masa de la razón entera; [...] aparece como exhortación a un actuar libre, la individualidad me es dada justamente a través de esa exhortación" ${ }^{3}$. Me es «dada», y por eso yo "me encuentro», lo cual manifiesta una dualidad de acción («me») y limitación («encuentro») y una identidad entre lo ideal y lo real: me pongo como acción originaria y a la vez me hallo individualizado y sensibilizado en un mundo. Yo me encuentro queriendo ${ }^{4}$, en un acto de querer libre, desde mí, no causado por algo exterior, pero sí propiciado o posibilitado por los otros seres racionales (la exhortación no es una causalidad mecánica, sino una petición). Esa es la dualidad con la que surge la conciencia empírica. Su acto de decidirse a querer algo concreto y determinado es identidad de lo real y de lo ideal, o sea, es una acción real (veremos que inteligible y sensible, nouménica y fenoménica a la vez) pero que sabe de sí, y para saber de sí tiene que contraponerse a lo otro, a los otros, y encontrarse entre ellos. Por consiguiente, esa acción de querer es sintética, o como dice Fichte es un pensar sintético (synthetisches Denken), en el cual se da a la vez una identidad y una diferencia entre sus elementos, pues síntesis es unión en la diferenciación, y gracias a eso es posible la conciencia. Por tanto en su reflexión (analítica) o conciencia reflexiva esa identidad se le aparece al Yo desdoblada en una serie o pensar ideal y en una serie o

\footnotetext{
${ }^{3}$ WLnm-K, GA IV/3, 470.

${ }^{4}$ De este dato parte la Ética de Fichte de 1798 en su $§ 1$, y lo analiza allí detenidamente.
} 
pensar real 5 . Veamos esos tres elementos de esta síntesis principal, cuyos miembros extremos se desdoblarán, de modo que la síntesis forma entonces un período de cinco momentos, lo que nos guiará hasta el final de la WLnm.

1) En el medio de este acto sintético, complejo, está la autodeterminación del individuo, solicitado o requerido o exhortado por el otro, a querer algo. Aquí sale a la superficie, a la conciencia del Yo, al ámbito de lo fenoménico, como querer empírico, lo que se había puesto al inicio del sistema como su primer principio y que como tal, como querer puro, no llegaría a la conciencia sensible, a saber, la acción, la agilidad, la libertad, en la forma de voluntad, identidad de ser y pensar, "porque su [del Yo] ser o esencia (Wesen) originaria, la que no puede ser ya deducida sino que ha de ser presupuesta en toda explicación, es un querer (Wollen) ${ }^{6}$. Precisamente porque no podemos deducirlo de un miembro superior sin destruirlo como libertad y como acción, es lo incomprensible (unbegreiflich), o sea, lo no captable mediante conceptos, que siempre ligan todo con otra cosa y lo hacen dependiente de ella: «este determinarse es el comienzo absoluto de toda vida y de toda conciencia, y justo por eso es incomprensible, porque toda conciencia siempre presupone algo ${ }^{7}$ como fundamento o principio de explicación para la comprensión de cualquier cosa. Por ello Fichte invita al principio a sus oyentes-lectores a intuir su propia acción, directamente. En consecuencia aquí el lenguaje flaquea, porque él además está atravesado en definitiva por lo sensible y la imaginación, y de suyo los términos de «mismidad absoluta, autonomía, libertad, todo eso es igual de incomprensible. La libertad sólo se deja describir negativamente, mediante un «no ser determinada»» ${ }^{8}$. Únicamente podemos describir la ley (de la génesis) por la que le debe surgir ese concepto al Yo,

\footnotetext{
${ }^{5}$ En otras filosofías sólo hay una serie, mientras que la DC atiende a la duplicidad necesaria para la conciencia (WLnm-K, GA IV/3, 495). Gracias a esa dualidad realidad-idealidad en el Yo, el filósofo idealista crítico o transcendental puede explicar la representación (Vorstellung) o saber, mientras que esto no lo logra el dogmático, que es incapaz de mostrar cómo se puede pasar del ser al saber (EE § 6, GA I/4, 195-199).

${ }^{6} \mathrm{WLnm}-\mathrm{K}, \mathrm{GA}$ IV/3, 498. «El concepto de exhortación no es lo primero, sino el querer» (o. c., 477). Véase también WLnm-H, GA IV/2, 230.

${ }^{7}$ WLnm-K, GA IV/3, 494.

${ }^{8}$ WLnm-K, GA IV/3, 498.

${ }_{9}$ «Todo lo espiritual es designado mediante expresiones sensibles; de ahí proceden muchos malentendidos, pues los signos son a menudo arbitrarios. Por ello, cuando se utiliza un signo, tiene que darse una explicación. Ahora bien, si debe darse una explicación allí donde falta la palabra,
} 
como hemos hecho ahora, e invitarle que lo lleve a cabo' . Este acto de autodeterminación (A) constituye el punto central de la síntesis, que se desarrollará finalmente en otros cuatro miembros, según el esquema de la quintuplicidad propio de Fichte: «Para facilitar la memorización vamos a llamar al punto medio de la síntesis A; lo real que se halla más próximo se denomina $\mathrm{B}$, lo real externo que se enlaza a ello G; del otro lado [en la serie ideal] llamaremos a lo que está más próximo $\beta$ y a lo exterior $\gamma{ }^{10}$, o sea, $\gamma \leftarrow \beta \leftarrow A \rightarrow B \rightarrow G$. Iremos viendo el significado de cada letra ${ }^{1}$.

2) La serie o pensar real es el pensamiento de un objeto real que debe ser producido por la causalidad del querer, del acto de querer del Yo-individuo: quiero, actúo y muevo mi mano, por ejemplo. El primer elemento de esta serie (B) es el cuerpo propio (Leib), deducido ya (\$§ 14 y 15) como siendo la voluntad vista en la forma imaginativa de la intuición espacio-temporal. Pero ahora, en este $§ 17$, se muestra además como el lugar donde el querer que se determina a la acción encuentra su causalidad efectiva (Wirksamkeit) en el mundo, a saber, en la fuerza sensible y física de su cuerpo. El Yo determinante y su concepto de fin (lo que él se propone hacer) se sensibiliza ahí y se hace fuerza real y causa, un pensar sensible: «mi cuerpo no es otra cosa que una cierta perspectiva (Ansicht) de mi causalidad como inteligencia» ${ }^{12}$. Él es la conciencia inmediata en la serie o pensar real ${ }^{13}$.

allí tiene que explicarse la cosa misma, esto es, tiene que explicarse genéticamente: yo me pongo y, en cuanto hago esto, observo que [338] lo hago de una cierta manera y que sólo puedo hacerlo así; puede suceder que todavía haya otras muchas cosas que sólo pueda hacer de esta manera, y entonces tengo una ley; por ello se habla de leyes del intuir, del pensar, etc.; este pensar necesario son leyes del pensar. Las leyes son propiamente sólo para un ser actuante, al que habitualmente consideramos como libre, pues decimos: tú tienes que proceder de tal o cual manera; análogamente decimos: el ser racional tiene que proceder de tal o cual manera, y éstas son sus leyes» (WLnm-K, GA IV/3, 337-338).

${ }^{10}$ WLnm-K, GA IV/3, 483. «Un PERIODUM sintético es siempre quíntuple. Aquí [en la deducción emprendida en la WLnm] tenemos la ventaja de ir desde los [miembros] internos hacia fuera, no como en la Doctrina de la Ciencia impresa [la $G W L]$ de fuera hacia adentro. Dentro de esta síntesis se halla todo pensar, pues todo es una determinada autoconciencia» (o. c., 477).

${ }^{11}$ Aquí atenderemos al significado que Fichte da a esas letras en la segunda mitad del § 17 (pp. 481 ss). Téngase en cuenta que en la primera mitad de ese parágrafo Fichte les asigna otro significado, e incluso utiliza en parte otras letras; pero esto ha sido explicado por el Prof. Carlos Morujão en su comentario.

${ }^{12}$ WLnm-K, GA IV/3, 484-485.

${ }^{13}$ «Tendríamos pues en la serie real ya dos cosas, la fuerza física y el sentimiento [...] La síntesis principal A consiste en la unión de lo ideal y lo real; lo real más cercano [al centro, a A] es 
El segundo miembro o conciencia mediada (mediada por el primer miembro descrito) de esta serie real (G) es el sentimiento producido en el Yo por dicha fuerza física, por cuanto que ahí el pensar está ligado o sujetado (gebunden) a algo objetivo; un sentimiento cuyo carácter estudiamos en el $\$ 6$ como momento en el cual el Yo toma conciencia de la limitación que le ocasiona la resistencia (§ 5) que encuentra su actividad real por parte del No-Yo. Ahora comprendemos que esa resistencia es ejercida contra la fuerza física de su cuerpo sentido (órgano externo) y articulado. De igual modo que antes pasábamos del sentimiento a la intuición del contenido de ese sentimiento (§ 7), aquí pasamos al objeto: de la fuerza sensible vamos al sentimiento, y de ahí el Yo intuye el objeto o acción objetiva y sensible que realiza mediante su querer. Por ejemplo, él mueve la mano porque así lo quiere según su concepto-fin y al mismo tiempo ve (intuye, percibe) cómo su mano se mueve objetivamente y cómo, por ejemplo, traslada un objeto según su propósito o bien lo produce. El Yo capta su objeto a través de su acción efectiva, de su hacer (Machen), de su causalidad: las letras que escribo, la estatua que esculpo ${ }^{14}$. En este momento el Yo sale al exterior, admite un mundo externo. Pero eso lo lleva a cabo por medio de la categoría de causalidad, apoyándose ciertamente en el sentimiento y la acción efectiva sensibilizada. En efecto, por medio del pensamiento, de la categoría de causalidad, añado el objeto al concepto de fin, añado un efecto a un concepto como concepto que produce un efecto. «Las categorías son los modos como la conciencia inmediata se convierte en conciencia mediata» ${ }^{15}$, aquí, gracias a la categoría de causa, la conciencia de la fuerza sensible del cuerpo se convierte en la conciencia mediada del objeto realizado. De esa manera el Yo sale de su mero pensarse a sí mismo al pensamiento y conocimiento de otras cosas distintas. Por eso las categorías son productivas (produzierend), aquí la de causalidad produce el objeto, claro que lo produce idealiter no ex nibilo, lo produce para el Yo, o sea, el Yo se entera del objeto producido por medio de esa categoría que se aplica a su fuerza sensible corporal y a su sentimiento y parte de ellos. La libertad no produce ese objeto $e x$ nibilo porque, como se verá en el $\$ 19$, su propio cuerpo como tal es un pro-

ahora la fuerza física del Yo mismo [...] Yo intuyo inmediata o directamente el acto de determinar mi fuerza física en el pensar el objeto» (WLnm-K, GA IV/3, 486).

${ }_{14}$ «El determinar o el concepto de fin del Yo debe contener el fundamente de la constitución o configuración (Beschaffenheit) del objeto. De esa manera viene al ánimo el principio del fundamento» (WLnm-K, GA IV/ , 483) o categoría de la causalidad.

${ }^{15}$ WLnm-K, GA IV/3, 485. 
ducto de la naturaleza y no de su acción libre; la libertad, el Yo, es una acción originaria pero finita, y ése sería el horizonte de la reflexión o filosofía transcendental. La categoría de causa es por tanto un elemento necesario en la configuración de la objetividad. Las otras dos categorías que pertenecen aquí y que veremos a continuación son la substancialidad y la acción recíproca. Ellas habían sido estudiadas por Kant como las tres categorías de relación.

3) En el acto de autodeterminación del querer del individuo (A) hay también una serie o pensar ideal. El primer elemento de esa serie ( $\beta$ ) es, como ya sabemos, el concepto de fin, que nos había aparecido por primera vez en el $§ 4$. Él es producido por el mero pensar; gracias a él el Yo formula su acto de querer y sabe lo que quiere ${ }^{16}$. En él me pienso como pensante, y su objeto es un querer. Pero ese concepto de fin, para tener causalidad efectiva, ha de sensibilizarse como fuerza de un cuerpo, de su cuerpo. Por una parte, el concepto de fin aparece como algo meramente ideal cuando es visto como procediendo del Yo inteligencia, pero también como algo real gracias a la fuerza física de nuestro cuerpo y su efectividad en el mundo sensible. El tema aquí es esta sensibilización del concepto de fin en la fuerza del cuerpo. Antes, en la serie o pensar real, veíamos cómo la fuerza física de nuestro cuerpo se sensibilizaba mediante el sentimiento objetivo y la realización del objeto querido. Ahora damos un paso atrás, hacia la fuente de la autodeterminación del Yo (A), y nos preguntamos cómo es posible que el Yo vea esa fuerza física como su fuerza; ésta es «la primera sensibilización» del pensar puro $^{17}$. Eso ocurre mediante el concepto de fin, viendo que la fuerza física actúa según lo requiere dicho concepto, conforme al propósito que tiene el Yo determinante: "esa fuerza física es la mía únicamente en la medida en que es contemplada a través del concepto de fin ${ }^{18}$. Pero ¿cómo se sensibiliza el concepto de fin en esa fuerza real? Estudiando este mecanismo o leyes de la conciencia veremos que, de igual manera que en la anterior sensibilización se añadía al Yo determinante el objeto sensible producido gracias a la categoría de causalidad, aquí se añadirá al Yo determinante el yo empírico, substante $(\gamma)$, en virtud de la categoría de substancia.

${ }^{16}$ Aunque pensamos que el contenido de mi querer está condicionado por la sociedad, por la propaganda, por la presión del grupo, por el miedo, etc, es decir, por factores externos, no es menos cierto que es el Yo el que tiene que hacerlo suyo de sí.

${ }^{17}$ WLnm-K, GA IV/3, 486.

${ }^{18}$ WLnm-K, GA IV/3, 486. 
En este proceso interviene la imaginación productiva ${ }^{19}$. Antes atendimos a la realidad física de la fuerza sensible del cuerpo como primer miembro de la serie real, mientras que aquí se ha de ver su idealidad (el que sea sensible, sentida por el Yo) para ligarla con el concepto de fin, que es asimismo una idealidad. Todos los elementos del sistema son mezcla de idealidad y realidad ${ }^{20}$, también la fuerza de nuestro cuerpo es a la vez real e ideal. Esa fuerza es ideal o subjetiva en cuanto está determinada por el concepto de fin, donde me sé activo, y eso tiene que ser intuido por el Yo, pues él ha de enterarse de ello. Pues bien, como ya hemos visto repetidas veces, sólo me puedo ver activo pasando de lo determinable a lo determinado. Lo determinado aquí es el concepto de fin, con el cual decido hacer esto y no lo otro. ¿ Y cómo se presenta lo determinable gracias al cual el Yo se ve y se intuye activo en el concepto de fin y en la fuerza física determinada por él? La respuesta de Fichte es que eso determinable procede de la imaginación productiva, que aquí ya no se circunscribe a configurar el objeto empírico, como en Kant, sino que también muestra en la intuición al sujeto-cuerpo empírico y temporal como facultad de querer o substancia espiritual (nos estamos fijando en su idealidad, en la serie ideal) capaz de decidirse y autodeterminarse.

En efecto, la imaginación, como ya nos lo había mostrado Kant, es síntesis originaria de lo diverso, capacidad de captar en unidad una multiplicidad, de sintetizar los opuestos (simultáneos o sucesivos) en un solo acto. Por eso ella puede comprender y hacer posible lo vivo y el movimiento en su unidad y en su multiplicidad (infinitamente divisibles después por el pensar, por el juicio) simultáneamente, es decir, el movimiento en cuanto una fuerza simple que se mueve y

19 «La cosa está así, en primer lugar hay un pensar puro que se determina; éste es visto en la síntesis por la imaginación y sensibilizado; en esta sensibilización, el mero concepto puro de fin se convierte en una determinación de una fuerza sensible, lo producido de ese modo [es] un objeto sensible; aquí en primer lugar hemos de ver en general, cómo la inteligencia llega a atribuirse una fuerza sensible, es decir, un cuerpo propio y una manifestación de fuerza del mismo, esta última no es otra cosa que el pensar puro visto únicamente a través de la imaginación» (WLnm-K, GA IV/3, 495).

${ }^{20}$ WLnm-K, GA IV/3, 486 y 496. "Así también aquí ideal y real, esos términos valen sólo relativamente. Hay ciertamente algo absolutamente ideal y algo absolutamente real, lo primero es lo inteligible y lo segundo la materia muerta. Pero los miembros que se halla entremedias pueden en un cierto respecto ser llamados ideales y en otro reales, según sean referidos hacia delante a lo absolutamente ideal o hacia atrás a lo absolutamente real» (WLnm-H, GA IV/2, 208). 
progresa como un continuo en una serie sucesiva de multiplicidades espacio-temporales, como cuando trazamos una línea y captamos la unidad de esa acción sin necesidad de atender a los infinitos puntos que se encuentran en ella, pues eso me detendría infinitamente y no me dejaría avanzar un paso. El pensar, por el contrario, para, fija, determina, y al querer pensar el movimiento se enreda en esos infinitos puntos, de manera que se le escapa como el agua entre los dedos; de ahí surgen las conocidas aporías de Zenón. Aplicando esto a nuestro caso, podemos decir que es la imaginación productiva la que convierte el acto puntual de autodeterminación del Yo (A) en un fluir o discurrir progresivo temporal (Flie en, Fortflie en $)^{2}$, lo cual permite al Yo intuirlo en el tiempo e intuirse de ese modo a sí mismo objetivamente como determinante, es decir, deja que el Yo mismo se muestre a sí, como activo, en su conciencia empírica. En el pensar hay determinación, un fijar y detener (lauter Stehen); el pensamiento decide, se autodetermina, mientras que la imaginación recorre y retiene en unidad viva y temporal esas diversas decisiones, y construye el determinable de ellas como un algo infinitamente divisible de posibilidades de acción del Yo. Es un proceso análogo a lo que podemos experimentar cuando deliberamos o dudamos y estamos todavía sin decidirnos qué cosa concreta querer, hasta que la decisión elimina esa oscilación y concentra nuestro pensar y toda su energía o fuerza en un solo punto ${ }^{22}$. En ese juego recíproco de pensamiento (determinación) e imaginación (nueva apertura y oscilación), en ese oscilar (Schweben) de la imaginación entre las distintas posibilidades de acción y las diversas decisiones o conceptos de fin o quereres particulares, ella construye un continuo fluir de una fuerza simple en el tiempo, la cual se manifiesta por tanto, por caer en el tiempo, como fuerza corporal. De esa unidad imaginativa, que flota y oscila entre opuestos, sale a la postre el pensamiento (para Fichte un pensamiento abstracto, algo meramente pensado, pues lo concreto es el efectivo acto de querer) de un ente sensible capaz de decidirse, y en ese sentido un ente ideal o espiritual, o sea, con facultad de querer (Vermögen zum Wollen). En ese marco se intuye cada decisión, situándola en un espacio y en un tiempo, y el Yo se hace consciente de ser lo activo en el establecimiento de su fin y en la orientación de su fuerza física; él aparece como el ente sensible-espiritual capaz de determinarse.

${ }^{21}$ WLnm-K, GA IV/3, 494, 500.

${ }^{22}$ WLnm-K, GA IV/3, 490, 501. «La imaginación no descansa en ninguna parte, ella va siempre de una cosa a otra. La determinación es en todos los sitios cerrada, y de nuevo abierta» (WLnmH, GA IV/2, 218). 
Aquí se produce una substancialización del Yo, que lo convierte en intuible, en empírico (la substancia es una categoría de lo fenoménico también en Kant), y que después aparecerá como un individuo perteneciente a la comunidad de seres racionales finitos. Eso ocurre porque ese fluir, o sea, lo sensibilizado por el acto de una imaginación siempre fluctuante y mediante el cual se temporaliza el pensar puro, es a su vez determinado y fijado por ese mismo pensar, captado como un todo. Entonces, gracias a esa determinación recíproca entre imaginación y pensar, el Yo como capacidad (Vermögen) deviene una substancia o quantum finito, algo determinado y sensible, algo fijo que dura en el tiempo (eso es la substancia fenoménica también en Kant), de modo que sus diversas decisiones aparecen ahora como sus accidentes que ocurren en diferentes momentos del tiempo $^{23}$. La causalidad sólo ofrecía simultaneidad entre la causa y el efecto (el efecto se produce cuando se da la causa), mientras que aquí el concepto de fin y la acción de esbozarlo adquiere duración, demandan un tiempo, de manera que se produce un actuar sensible sucesivo y un progresivo surgimiento del producto de nuestro actuar ${ }^{24}$. Eso es lo que se añade a la autodeterminación del Yo y a su concepto de fin por medio de la categoría de substancialidad (por medio del pensar), que es también productiva. A la conciencia inmediata del concepto de fin $(\beta)$ se añade la conciencia mediatizada de un Yo substante $(\gamma)$, que dura en el tiempo, y con poder o facultad de querer, de pensar y de sentir, provisto de fuerza sensible, la cual no es sino el pensar puro visto desde la imaginación: «a esa sensibilización se conecta todo el mundo ${ }^{25}$. Así, sobre el fondo de ese determinable, percibo mi autodeterminación y mi causalidad como mía, pues en la categoría de substancia no nos fijamos en el producto (esto era lo que sucedía en la categoría de causa), sino en la subjetividad o idealidad de la acción. El Yo se substancializa, se sensibiliza, se hace fenómeno, surge para sí (para su conciencia)

\footnotetext{
${ }^{23}$ «Substancia es una mera capacidad o facultad o poder (Vermögen) que es encerrado entre límites, pero facultad lo es sólo en la medida en que es intuida por la imaginación; es una facultad limitada en la medida en que aquel construir de la imaginación es determinado por el pensar puro [...] de esa manera surge para mí ahora una fuerza que posee una facultad para una multiplicidad infinita [de acciones y decisiones]» (WLnm-K, GA IV/3, 502).

${ }^{24}$ «La causa y el efecto son simultáneos, por medio del concepto de causalidad no surge ningún tiempo, en la naturaleza no surge por tanto el tiempo, el tiempo surge únicamente en el Yo, en el concepto de la substancialidad aplicado al Yo, en el recorrido que hace la imaginación de las posibilidades de acción; por el hecho de que el objeto es mero objeto del Yo que actúa, el objeto es entonces extendido también a través del tiempo» (WLnm-K, GA IV/3, 506-507).

${ }^{25}$ WLnm-K, GA IV(3, 495.
} 
como algo, como un ser del mundo que quiere y piensa, como una substancia espiritual (espíritu significa capacidad de autodeterminación), que es intuida bajo la forma de la intuición interna (alma) y bajo la interna y la externa a la vez (cuerpo propio). Lo determinable aparece siempre como algo dado, aquí en concreto dado al acto de determinarse, y por tanto con objetividad, de manera que ese Yo substante se muestra como un yo efectivamente real (wirkliches Ich) ${ }^{26}$.

4) Por esta acción recíproca (Wechselwirkung) del intuir y del pensar «surge una facultad cerrada [fija, substante, finita] del Yo como fuerza sensible y una determinidad [o capacidad de ser determinada] de la misma (concepto de substancialidad), y a la determinidad de esa fuerza sensible se le añade con el pensamiento [en virtud de la categoría] un objeto que es determinado por ella en el pensamiento (concepto de causalidad) ${ }^{27}$. La voluntad-substancia no sería nada si no ejerciera una causalidad efectiva en el mundo, y el Yo no se encontraría queriendo, no se construiría como Yo, no sabría de sí. Y a la inversa, la causalidad del Yo no tendría lugar si éste no se sensibilizara desde sí en la fuerza física de su cuerpo. Todos los elementos que hemos visto: autodeterminación o querer, cuerpo propio, sentimiento y objeto producido, concepto de fin y sujeto empírico, son elementos o momentos de un mismo pensar sintético. Los dos puntos de vista, el de la causalidad y el de la substancialidad, el pensar real y el pensar ideal, se unifican (sin confundirse, pues entonces no habría distinción y por tanto tampoco conciencia, lo cual justifica y hace necesario el análisis de cada uno de esos elementos ${ }^{28}$ ) y se subordinan a la categoría de la relación recíproca. En esta, lo que mediatiza ya no es distinto de lo mediatizado, como ocurría en las otras dos categorías, sino que todo elemento es a la vez mediador de los demás y mediado por ellos, formando un todo orgánico, que nos conducirá al reino de los seres racionales desde la serie ideal, y al reino de la naturaleza desde la serie real.

En los parágrafos siguientes, $§ \S 18$ y 19, esta síntesis quíntuple aquí establecida en el individuo volente y actuante se abrirá justamente a esos dos ámbitos de totalidad, el de la naturaleza y el de la razón, estableciéndose una nueva y

${ }^{26}$ WLnm-K, GA IV/3, 491.

27 WLnm-K, GA IV/3, 498.

${ }^{28}$ «En la unificación se separan, y por la separación se unifican, ambas cosas son inseparables» (WLnm-K, GA IV/3, 475). «El inicio de toda conciencia es síntesis y análisis a la vez, y por este último surge un múltiple» (o. c., 476). 
más amplia síntesis (con pasos intermedios que se irán deduciendo), pues la WL «no contiene más que síntesis» ${ }^{29}$ y síntesis de síntesis. Allí los dos elementos de la serie real que aparecen en este $\$ 17$ ( $\mathrm{B}$ y $\mathrm{G}$ ) se contraen en uno solo, en $\mathrm{B}^{30}$, y se nos dice que en el $\$ 17$ únicamente habíamos examinado tres miembros de la quintuplicidad ${ }^{31}$, de manera que el término «G» cobrará un nuevo sentido y significará la totalidad de la naturaleza, en la que se inserta el cuerpo del Yo individuo y el objeto producido por su fuerza física sensible. Y eso mismo ocurre con los dos elementos de la serie ideal, pues $\gamma$ representará ahora la comunidad de todos los seres libres ligados por la exhortación (la educación, la organización socio-política, la cultura, la historia, la moral, la religión). De esa manera los dos miembros extremos de la quintuplicidad deducida en el $\$ 17$ se insertan en su determinable desde donde son también comprendidos, a saber, el objeto empírico (lo producido por la fuerza sensible del cuerpo conforme lo indicado en el concepto de fin) halla su lugar en el mundo sensible como totalidad o naturaleza existente sin mi intervención (lo determinable), y el Yo-individuo substante y empírico, donde el espíritu aparece como algo determinado, se comprende a partir de su determinable como un miembro de la comunidad de seres racionales y sensibles. La síntesis se completa en el $§ 19$ con la exposición de la relación recíproca de ambos ámbitos de la naturaleza y la razón y de su punto de unión en el cuerpo (Leib) articulado, mostrándose así la conciencia como un círculo ${ }^{32}$.

${ }^{29}$ WLnm-K, GA IV/3, 495.

${ }^{30}$ «Mi efectividad y a consecuencia de ésta un producto de la misma = B» (WLnm-H, GA IV/2, 238).

${ }^{31}$ WLnm-K, GA IV/3, 500, 502.

${ }^{32}$ WLnm-K, GA IV/3, 509. 